\title{
Viewpoint
}

\section{Geode: crystal-containing cavity, lymph space, or pseudocyst?}

\author{
D L GARDNER
}

From the Department of Histopathology, University Hospital of South Manchester, Nell Lane, Manchester $M 208 L R$

'An aphorism - - - has been forming in one of the blank interior spaces of my intelligence, like a crystal in the cavity of a geode.'

(Oliver Wendell Holmes (1860) in: The professor at the breakfast table.)

Misnomers and malapropisms are inevitable and commonplace in the early, observational phases of young sciences as investigators search for apt terms to describe new observations. When it is proposed to use an old word to define a new phenomenon, the proposal should not imply permanent, unquestioning acceptance. It may be time to re-examine some of the words that have been introduced into rheumatology and to determine whether they offer the best solutions to problems of nomenclature. Indeed, few would now regard 'rheumatism' as the ideal collective noun for the local and systemic diseases of articular and connective tissue.

\section{Geode}

The Oxford English Dictionary $(O E D)^{1}$ states that a geode is a 'a concretionary of nodular stone, containing a cavity usually lined with crystals or other mineral matter'; or 'the cavity itself, together with the crystal or mineral formation therein contained'. The word derives from the Greek for 'earth' and 'earthy'.

In contrast, Butterworths Medical Dictionary ${ }^{2}$ curiously defines a geode as 'any one of the minute tissue spaces which contain lymph, or any cavity related to the lymphatic system in general', and Dorland's Illustrated Medical Dictionary ${ }^{3}$ states that a geode is 'a dilated lymph space'.

The Shorter Oxford English Dictionary ${ }^{4}$ follows the approach chosen by the $O E D$, and Chambers

Accepted for publication 1 May 1985.
Dictionary of Science and Technology $y^{5}$ adopts essentially the same tactic.

Difficulties arise when modern texts on rheumatology are consulted. Following the usage of Dixon and Jayson ${ }^{6}$ Copeman's Textbook of the Rheumatic Diseases $^{7}$ uses 'geode' to describe the so-called pseudocysts that are one characteristic of the processes that destroy juxta-articular bone in rheumatoid arthritis. These pseudocysts ${ }^{8}$ closely resemble those of osteoarthrosis. In both disorders the pseudocyst replaces cancellous bone, in both the loss of bone signifies $x$-radiotranslucency, and in both the pseudocyst is formed of loose and often vascular, non-mineralised connective tissue having a 'mucoid' or 'myxoid' structure. The pseudocyst is not a cavity, it is not a nodular stone or concretion, and it does not contain or acquire crystalline deposits. Kelley's Textbook of Rheumatology and McCarty, editing Hollander's textbook, ${ }^{10}$ do not index geode.

Are we justified, therefore, in continuing to apply this old geological word to describe an island of loose connective tissue in the bone of rheumatoid arthritis? Probably not: a better and more precise descriptive term is needed. Among those that spring to mind are:

1. (rheumatoid) bone connective tissue island or node (or connective tissue node of bone);

2. (rheumatoid) myxoid node of bone;

3. (rheumatoid) osteoid cyst or pseudocyst;

4. (rheumatoid) osteomyxoid cyst or pseudocyst;

5. (rheumatoid) mucoid cyst or pseudocyst.

Of these, the description 'myxoid (rheumatoid) 
pseudocyst' seems to reflect the nature of the structure most accurately.

\section{References}

1 Oxford English Dictionary. 1933.

2 Butterworth's Medical Dictionary. 2nd ed. 1978.

3 Dorland's Illustrated Medical Dictionary. 25th ed. 1974.

4 Shorter Oxford English Dictionary. 1978.

5 Chambers Dictionary of Science and Technology. 1975.

6 Jayson M I V, Rubinstein D, Dixon A St J. Intra-articular pressure and rheumatoid geodes (bone 'cysts') Ann Rheum Dis 1970; 25: 496-502.

7 Scott J T (ed.). Copeman's textbook of the rheumatic diseases. 5th ed. Edinburgh-London-New York: Churchill Livingstone, 1978.

8 Cruickshank B, Macleod J G, Shearer W S. Subarticular pseudocysts in rheumatoid arthritis. Journal of the Faculty of $\frac{S}{D}$ Radiologists (London) 1954; 5: 218-26.

9 Kelley W N, Harris E D, Ruddy S, Sledge C B. Textbook of rheumatology. 1st ed. Philadelphia-London-Toronto: Saun- की ders, 1981.

10 McCarty D J (ed.). Arthritis and allied conditions. A textbook of rheumatology. 9th ed. Philadelphia: Lea and Febiger, 1979.

\section{Correspondence}

\section{Lyme disease in Italy: first reported case}

SIR, Since the first description of Lyme arthritis in $1976,{ }^{1}$ several cases have been reported in Europe (Fig. 1). Case reports from Switzerland and France have been published. ${ }^{2-5}$ Moreover, Ryberg and coworkers ${ }^{6}$ suggested a close aetiological relationship between European lymphocytic meningoradiculitis, a well known neurological disorder first delineated by Bannwarth, ${ }^{7}$ and Lyme arthritis.

We report the first case of Lyme arthritis observed in Italy. In July 1983 a middle aged woman, a farmer in the neighbourhood of Genoa, developed on her thighs the classical erythema chronicum migrans (ECM) lesions after a tick bite: two erythematous circular patches (diameter 30 $\mathrm{cm}$ ) with smooth surfaces and absence of scaling surrounded by a red band, thus resembling a target. After a few days she complained of malaise, fever, stiff neck, and intense pain at the site of the ECM lesions; a general practitioner who suspected a rickettsial infection endemic in that area prescribed tetracycline. In August after clearing of the skin lesions her knees and hips became painful. On examination low back symptoms were predominant with a positive right straight leg raising test at $60^{\circ}$ and diminished right knee reflex; limited painful neck movements suggested a polyneuroradiculopathy. Serum tests, including a search for cryoglobulins and immune complexes (Clq binding assay), were negative. One month later (September 1983) she developed an oligoarthritis of the metacarpophalangeal and proximal interphalangeal joints of the right index finger, that promptly resolved on non-steroidal anti-inflammatory therapy. By immunofluorescent assay ${ }^{8}$ high titres (1/512) of IgG antibodies against the Lyme spirochaete were detected in a serum sample collected 6 weeks after the bite, thus confirming the clinical diagnosis. In summer 1984 when checked for late manifestations of the disease the patient was completely asymptomatic and refused further blood sample collection.
For this reason we could not follow the progress of the antibody response.

It is noteworthy that in our case, in accordance with $\frac{C}{c}$ Steere and coworkers, ${ }^{9}$ the prompt use of antibiotic $\overrightarrow{\vec{\theta}}$ therapy slowed the clinical manifestations of the disease, $\stackrel{\infty}{\infty}$ but nevertheless the immune response was very intense. of

As expected, because of the wide distribution of Ixodes ricinus in Europe and the presence of the disease in bordering countries, Lyme disease is present also in Italy. New cases will be recognised probably in the near future in Italy and in other European countries. The question $\frac{\mathbb{D}}{8}$

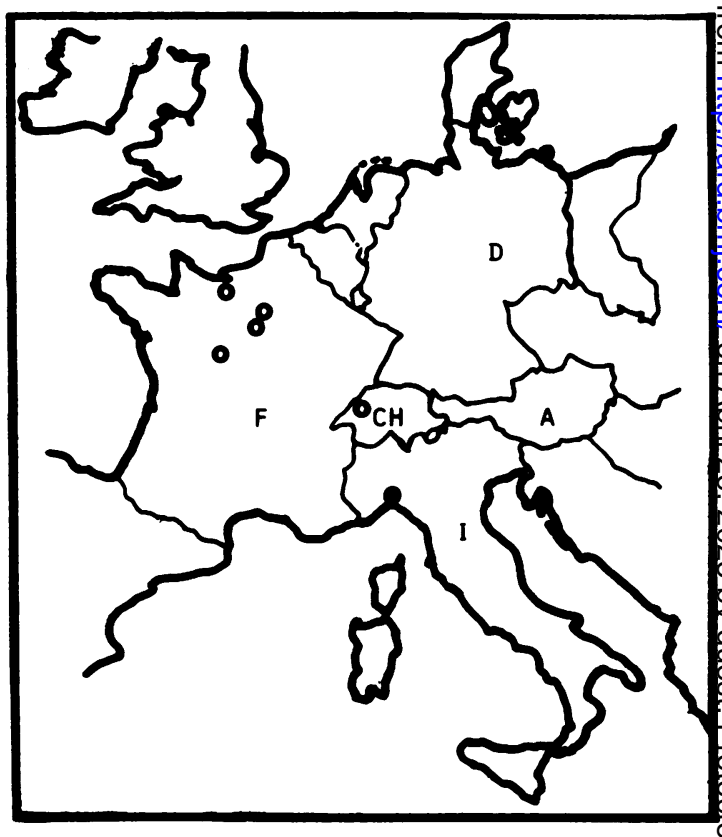

Fig. 1 Our (full circle) and other (open circles) cases of Lyme disease observed in Europe: geographic distribution. 